\title{
Eleven COVID-19 Outbreaks with Local Transmissions Caused by the Imported SARS-CoV-2 Delta VOC — China, July-August, 2021
}

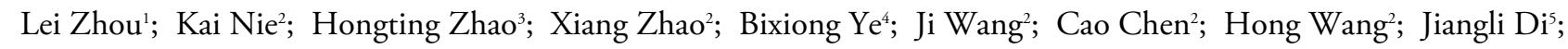

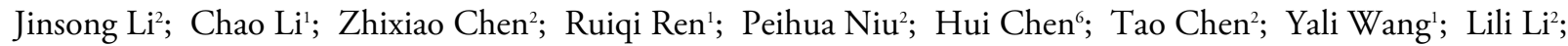

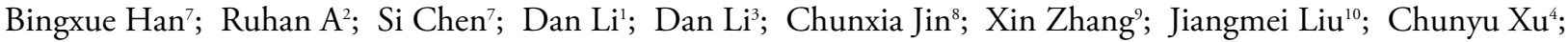
Yecheng $\mathrm{Yao}^{11}$; Yenan Feng'; Zhili $\mathrm{Li}^{3}$; Bike Zhang ${ }^{12}$; Yang Song'; Peter $\mathrm{Hao}^{12}$; Yanping Zhang'; Hao ${ }^{12}{ }^{12}$;

Qun $\mathrm{Li}^{1}$; George F. Gao ${ }^{12}$; Guoqing Shi ${ }^{1, *}$; Wenbo $\mathrm{Xu}^{2, *}$

Starting from December 2019, Wuhan, China, encountered the first outbreak of coronavirus disease 2019 (COVID-19) (1-2). The epidemic was successfully suppressed by strict containment so that the number of infected people was reduced to 0 on April 8, 2020 (3-4). After that, China experienced roughly 3 dozen outbreaks with local transmission caused by imported severe acute respiratory syndrome coronavirus 2 (SARS-CoV-2). These outbreaks were then contained by an effective suppression strategy, and the number of infected people has successfully reached zero again. However, local outbreaks of COVID-19 have reappeared in several areas in China recently and were caused by the SARS-CoV-2 Delta variant of concern (VOC) (5).

As of August 26, 2021, a total of 1,390 cases of COVID-19 had been reported in 50 cities in 19 provincial-level administrative divisions (PLADs) (Figure 1), all of which were found to involve the Delta variant.

Furthermore, these cases were found to stem from 12 genetically distinct Delta variant imported sources that were grouped into 11 related outbreaks or sporadic case incidents, resulting in 10 geographically separated epidemics including the following areas (Figure 1): Nanjing City of Jiangsu Province; Zhengzhou City of Henan Province; Xiamen City of Fujian Province; Wuxi City of Jiangsu Province; Dehong City of Yunnan Province; Pudong District of Shanghai Municipality; Songjiang District of Shanghai Municipality; Haikou City of Hainan Province; Ningbo City of Zhejiang Province; and Bortala Prefecture of Xinjiang Uygur Autonomous Region.

Starting from July 20, 2021, the number of daily reported infections increased gradually but reached a peak reporting period from July 31 to August 11, 2021. During this peak period, the number of everyday reported cases exceeded 50, and a total of 969 cases were reported during this period, which comprised $69.7 \%$ of all cases reported during this series of epidemics. After August 12, the number of daily reported cases decreased significantly.

\section{INVESTIGATION AND RESULTS}

The spatial distribution of the cases was shown in Figure 1. The 5 areas with the highest cumulative reported cases were as follows: Yangzhou City of Jiangsu Province (570 cases, 41.0\%), Nanjing City of Jiangsu Province (235 cases, 16.9\%), Zhengzhou City of Henan Province $(139,10.0 \%)$, Wuhan City of Hubei Province (88 cases, 6.3\%), and Zhangjiajie City of Hunan Province (76 cases, 5.5\%). There were 1,390 cases reported in total, $108(7.8 \%)$ were asymptomatic cases and 1,282 (92.2\%) were confirmed symptomatic infections; $660(47.5 \%)$ cases occurred in males; and the median age of the patients was 44 years (interquartile range: 29-58). Populations of all age groups were affected, with most cases occurring in patients aged 20-70 years old. The median age of confirmed and symptomatic infections (45 years) and asymptomatic infections (32 years) differed significantly $(P<0.05)$. The occupational categories that comprised the highest proportions and exceeded $10 \%$ of the total were as follows: homemakers and unemployed persons (16.0\%), retired persons (13.3\%), laborers $(13.3 \%)$, farmers $(11.1 \%)$, public venue attendants $(10.8 \%)$, and students $(10.2 \%)$. More data were available in Table 1.

\section{Nanjing City Epidemic}

Of this series of epidemics, the epidemic in Nanjing City affected the largest geographical area and had the most significant cumulative number of cases. The investigation revealed that the index case, which was 


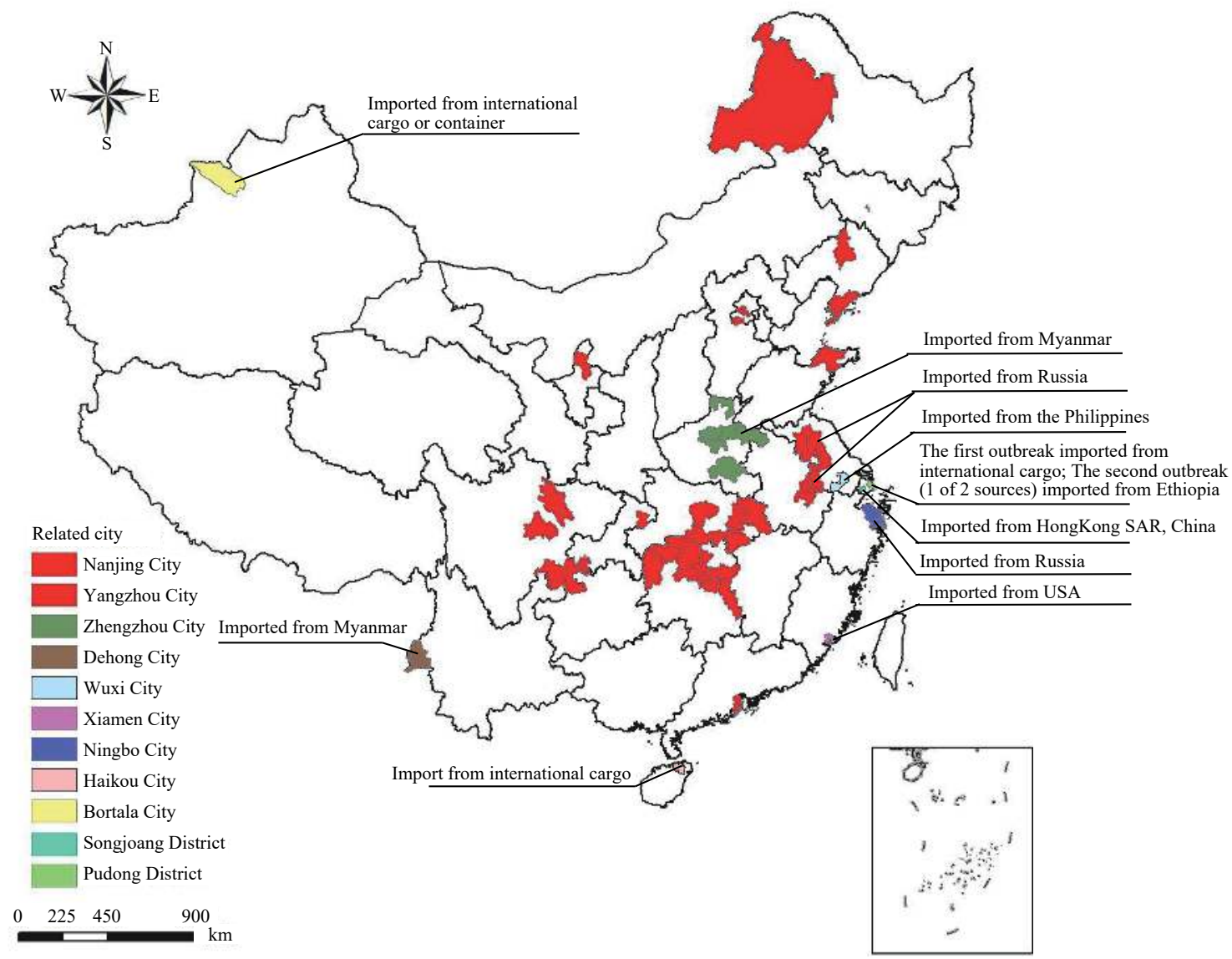

FIGURE 1. Spatial distribution of reported cases in the series of COVID-19 epidemics caused by the Delta variant in China from July 20 to August 26, 2021.

reported on July 20, 2021 most likely happened in a cabin cleaning staff member servicing Air China Flight CA910 from Moscow Sheremetyevo International Airport (SVO) to Nanjing Lukou Airport (NKG) on July 10. The genome of the strain of Delta VOC detected in the index case was identical to the genome of the strain isolated from an imported patient found in a passenger of the flight, who was tested while in mandatory two-week quarantine. Furthermore, this identical strain was detected in several other local cases in Nanjing in the days after the index case was found.

After the virus was introduced to airport staff through the cleaning staff member, it most likely first spread to other cleaning staff members and then to airport passengers, local communities, and families. This resulted in further community transmission, eventually spilling over to other cities and provinces.

From July 20 to 29, 2021, the reported infections in this epidemic were mostly limited to Nanjing. However, starting on July 30, the proportion of reported infections in neighboring Yangzhou City gradually exceeded that of Nanjing. As of August 26, 2021, this epidemic resulted in a total of 1,162 reported infections across 38 cities in 14 PLADs. The 5 areas with the highest number of reported illnesses were as follows: Yangzhou City (570 cases), Nanjing City (235 cases), Wuhan City (88 cases), Zhangjiajie City (76 cases), and Jingmen City (58 cases). Nanjing Lukou Airport was fully closed for prevention and control measures starting on July 26.

\section{Zhengzhou City Epidemic}

The first reported cases of the Zhengzhou City epidemic occurred in a pregnant woman and a cleaning staff member in the Sixth People's Hospital of Zhengzhou. After the cases were reported on July 31, all hospital-related personnel, including staff members, patients, and accompanying persons, immediately underwent nucleic acid testing, which yielded a total of 32 infection cases that were found that day. The index case was most likely in an accompanying person visiting a patient in the Tuberculosis 
TABLE 1. Demographic data for the series of COVID-19 epidemics caused by the B.1.617.2 (Delta) variant in China from July 20 to August 26,2021

\begin{tabular}{|c|c|c|c|c|}
\hline Characteristics & Total, n (\%) & $\begin{array}{c}\text { Asymptomatic } \\
\text { infections, } \mathrm{n}(\%)\end{array}$ & $\begin{array}{l}\text { Confirmed, symptomatic } \\
\text { infections, } \mathrm{n}(\%)\end{array}$ & $P$-value \\
\hline \multicolumn{5}{|l|}{ Sex } \\
\hline Male & $67(10.2)$ & $660(47.5)$ & $593(89.8)$ & $<0.05$ \\
\hline Female & $41(5.6)$ & $730(52.5)$ & $689(94.4)$ & \\
\hline \multicolumn{5}{|l|}{ Age } \\
\hline Mean \pm standard deviation & $32.0 \pm 17.8$ & $43.1 \pm 20.8$ & $44.1 \pm 20.7$ & $<0.05$ \\
\hline Median (interquartile range) & $32(17-46)$ & $44(29-58)$ & $45(30-59)$ & \\
\hline Range (minimum-maximum) & $1-77$ & $0-91$ & $0-91$ & \\
\hline$<18$ years old & $28(14.4)$ & $194(14.0)$ & $166(85.6)$ & $<0.05$ \\
\hline $18-59$ years old & $75(8.6)$ & $873(62.8)$ & $798(91.4)$ & \\
\hline$\geq 60$ years old & $5(1.5)$ & $323(23.2)$ & $318(98.5)$ & \\
\hline \multicolumn{5}{|l|}{ Occupation } \\
\hline Homemakers and unemployed persons & $9(4)$ & $223(16.0)$ & $214(96)$ & $<0.05$ \\
\hline Retirees & $2(1.1)$ & $185(13.3)$ & $183(98.9)$ & \\
\hline Worker & $28(15.1)$ & $185(13.3)$ & $157(84.9)$ & \\
\hline Farmer & $5(3.2)$ & $154(11.1)$ & $149(96.8)$ & \\
\hline Public venue attendant & $9(6)$ & $150(10.8)$ & $141(94)$ & \\
\hline Student & $23(16.2)$ & $142(10.2)$ & $119(83.8)$ & \\
\hline Public staff & $7(10)$ & $70(5.0)$ & $63(90)$ & \\
\hline Toddler/child & $7(12.1)$ & $58(4.2)$ & $51(87.9)$ & \\
\hline Sanitation staff & $1(1.9)$ & $53(3.8)$ & $52(98.1)$ & \\
\hline Medical staff & $3(8.1)$ & $37(2.7)$ & $34(91.9)$ & \\
\hline Catering industry & $1(5.9)$ & $17(1.2)$ & $16(94.1)$ & \\
\hline Teacher & $3(17.6)$ & $17(1.2)$ & $14(82.4)$ & \\
\hline Driver & $0(0)$ & $11(0.8)$ & $11(100)$ & \\
\hline Other & $10(11.4)$ & $88(6.3)$ & $78(88.6)$ & \\
\hline \multicolumn{5}{|l|}{ Epidemic center } \\
\hline Nanjing, Jiangsu & $101(8.7)$ & $1,162(83.6)$ & $1,061(91.3)$ & $<0.05$ \\
\hline Zhengzhou, Henan & $1(0.6)$ & $167(12.0)$ & $166(99.4)$ & \\
\hline 9 other outbreaks & $6(9.8)$ & $61(4.4)$ & $55(90.2)$ & \\
\hline Total & $108(7.8)$ & $1,390(100)$ & $1,282(92.2)$ & \\
\hline
\end{tabular}

Complications Department of the Sixth People's Hospital of Zhengzhou; this accompanying person started experiencing symptoms on July 24.

Previously, the Sixth People's Hospital of Zhengzhou admitted two imported cases of COVID19 from the Yangon City of Myanmar. The cases occurred in persons entering China in early July and were admitted to the hospital for isolation treatment. Since the isolation ward for COVID-19 at the hospital was adjacent to $\mathrm{TB}$ ward, the transmission was suspected to occur that first infected attendants and patients of the department, then spread through the rest of the hospital through close contact with other medical staff, cleaning staff, accompanying persons, and other populations. Afterward, COVID-19 likely spilled out to the rest of Zhengzhou and 5 other cities in Henan Province.

As of August 26, a total of 167 cases have been linked to this epidemic that stemmed from the Sixth People's Hospital of Zhengzhou. The genetic sequence of the first reported case, the likely index case, and the imported cases from Myanmar were homologous (Figure 2), so the imported cases were the possible source of this epidemic. The Sixth People's Hospital of 


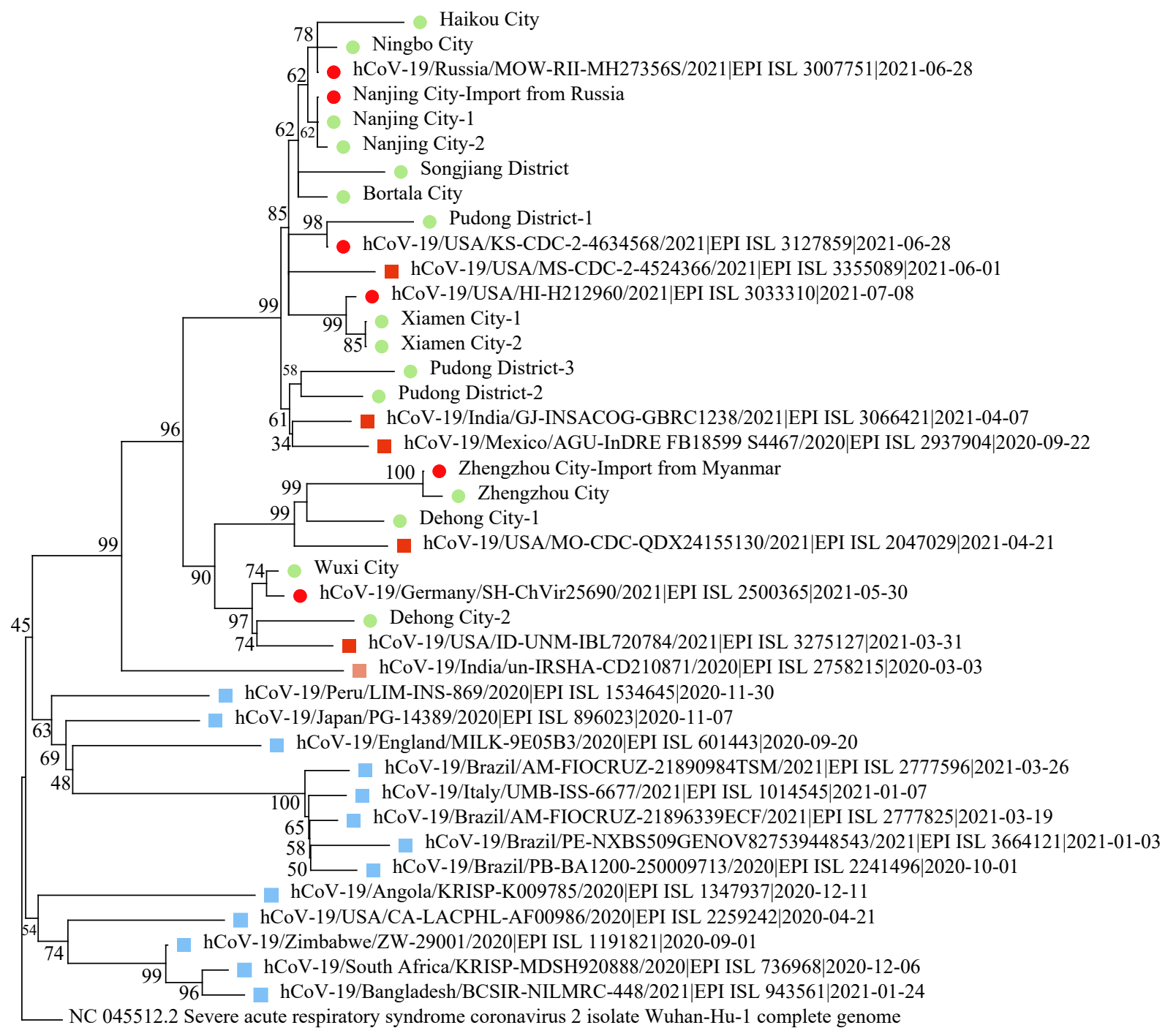

$\stackrel{\longmapsto}{0.00020}$

FIGURE 2. Phylogenetic analysis of SARS-CoV-2 based on full-length genome sequences.

Note: The Delta variants that caused the eleven recent outbreaks in China are indicated by green dots, and the Delta variants of imported case (or GISAID) with the highest genome homology to the local epidemic strains, the Delta variants from outside China (including lineages B.1.617.2, AY.1, AY.2, AY.3, and AY.3.1), the Kappa variants (lineage B.1.617.1), and the other VOCs and VOls are indicated by red dots, orange squares, light orange square, and blue squares, respectively. The tree was rooted using the prototype strain isolated in Wuhan. A neighbour-joining phylogenetic tree was constructed using MEGA (v7.0), and the Kimura 2-parameter model with 1,000 bootstrap replicates was used.

Zhengzhou and its neighboring communities were closed for prevention and control measures starting on July 31.

\section{Other Epidemics}

Since July 20, 2021, 9 other smaller-scale epidemics/outbreaks have been reported and investigated in other parts of the country; all 9 outbreaks were suspected originated from imported cases or cargos contaminated by SARS-CoV-2. As of
August 26, a total of 61 cases had been reported in the following areas: Dehong City of Yunnan Province (40 cases); Xiamen City of Fujian Province (5 cases); Wuxi City of Jiangsu Province (1 case); Pudong District of Shanghai Municipality (8 cases, from three independent sources); Songjiang District of Shanghai Municipality (2 cases); Haikou City of Hainan Province (1 case); Ningbo City of Zhejiang Province (1 case); and Bortala Prefecture of Xinjiang Uygur Autonomous Region (3 cases). 
The resulting investigation yielded a variety of causes. The Dehong Epidemic was most likely due to imported cases in individuals entering China through the land border with Myanmar. The Xiamen Outbreak was most likely caused by pilots of international cargo flights from the United States. The source of the Delta variant in the sporadic cases incidents in Wuxi and Songjiang District were related to imported cases in local designated hospitals. The source of the first outbreak in Pudong was most likely due to a ground crewmember of Pudong Airport shuttle crews for international flights. The source of the sporadic cases in Haikou and Ningbo and the second outbreak in Pudong were most likely to be spread by international cargo porters in the airports of or ports the three cities. The Bortala Prefecture Outbreak was probably due to transmission by international cargo or container. Genetic sequencing (Figure 2), molecular epidemiological analysis, and epidemiological methods indicated the source of the Delta variant of the 7 COVID-19 epidemics/outbreaks were linked to the following countries/areas: the Dehong outbreak was related to imported cases from Myanmar; the Nanjing outbreak from Russia; the Wuxi outbreak from the Philippines; the Zhengzhou outbreak from Myanmar; the Xiamen outbreak from the United States; the Ningbo outbreak from Russia; and the second Pudong District outbreak (one of the two sources) from Ethiopia; the Songjiang District outbreak from HongKong SAR, China. No highly homologous genome sequences were found of the other three outbreaks related to the Delta variant (Xinjiang, Haikou, and one of the two Pudong oubreaks in Shanghai) among the SARS-CoV-2 database of domestic and on Global Initiative on Sharing Avian Influenza Data-EpiCoV (GISAID-EpiCoV) platform (https://www.gisaid.org). The detected strains circulated in different periods but were closely related.

\section{PUBLIC HEALTH RESPONSE}

Following the emergence of the epidemics, provincial authorities initiated an emergency response, and the National Health Commission (NHC) immediately formed a working group that included experts from China CDC and delegated them to Nanjing, Zhangjiajie, Yangzhou, Zhengzhou, Wuhan, and other affected areas to provide onsite guidance. Several affected venues, including Nanjing Lukou Airport and the Sixth People's Hospital of Zhengzhou, were closed to respond to the epidemic and reduce transmission through prevention and control measures.

Prevention and control of these epidemics were still based on the previous strategies and measures ( 6 ). Risk areas were delineated, at-risk people and close contacts were determined and tracked, large-scale population nucleic acid screening was implemented, and detection and management of the sources of infection were completed as early as possible. Responsible societal stakeholders were mobilized to encourage compliance and cooperation by fully utilizing existing communityled grassroots grid management systems.

\section{DISCUSSION}

The sources of SARS-CoV-2 infections for several epidemics inside China after the wave of Wuhan outbreak have been found previously. For instance, imported cases and related transmission were caused by flights (7), carried by crewmembers (8), carried by community members (9), and spread in hospital settings (10). Increasing awareness and remaining vigilant will help prepare the necessary emergency response resources and prevent the uncontrolled spread of COVID-19.

The measures utilized in these responses and other strategies helped terminate community transmission, strengthen infection control, and prevented spread in critical venues such as medical institutions, designated patient isolation facilities, and nursing homes. As is known, the transmission of Delta variant was even faster and more severe than the prototype strain that was isolated in Wuhan (5); in order to ensure the suppression of this virus, the time period for considering a contact to be a close contact was lengthened to increase the sensitivity of close contact identification to find infected individuals. Furthermore, the timely release of information and communication of risks allowed for heightened public awareness and, when coupled with improved health education for the public, helped achieve better use of emergency response resources and better outcomes for the epidemic prevention and control strategies. Through careful implementation of various active and effective measures, the number of cases in this series of epidemics has been reduced to zero.

Funding: Supported by the National Natural Science Foundation (grant number 71934002) of the People's Republic of China.

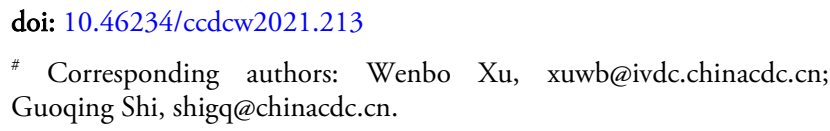




\begin{abstract}
1 Public Health Emergency Center, Chinese Center for Disease Control and Prevention, Beijing, China; ${ }^{2}$ NHC key laboratory for Medical Virology, National Institute for Viral Disease Control and Prevention, Chinese Center for Disease Control and Prevention, Beijing, China; ${ }^{3}$ Division of Infectious Disease, Key Laboratory of Infectious Disease Surveillance and Ear-warning, Chinese Center for Disease Control and Prevention, Beijing, China; ${ }^{4}$ National Institute of Environmental Health, Chinese Center for Disease Control and Prevention, Beijing, China; ${ }^{5}$ National Center for Women and Children's Health, Chinese Center for Disease Control and Prevention, Beijing, China; ${ }^{6}$ National Center for Tuberculosis Control and Prevention, Chinese Center for Disease Control and Prevention, Beijing, China; ${ }^{7}$ Weifang Medical University, Weifang, Shandong, China; ${ }^{8}$ Anti-plague Institute of Hebei Province, Zhangjiakou, Hebei, China;

${ }^{9}$ The First Institue for Endemic Diseases Control and Prevention of Jilin Province, Baicheng, Jilin, China; ${ }^{10}$ National Center for Chronic and Noncommunicable Disease Control and Prevention, Chinese Center for Disease Control and Prevention, Beijing, China; ${ }^{11}$ National Institute for Nutrition and Health, Chinese Center for Disease Control and Prevention, Beijing, China; ${ }^{12}$ Chinese Center for Disease Control and Prevention, Beijing, China.
\end{abstract}

Submitted: September 23, 2021; Accepted: October 01, 2021

\section{REFERENCES}

1. Tan WJ, Zhao X, Ma XJ, Wang WL, Niu PH, Xu WB, et al. A Novel coronavirus genome identified in a cluster of pneumonia cases-Wuhan, China 2019-2020. China CDC Wkly 2020;2(4):61 2. http://dx.doi.org/10.46234/ccdcw2020.017.

2. Zhu N, Zhang DY, Wang WL, Li XW, Yang B, Song JD, et al. A novel coronavirus from patients with pneumonia in China, 2019. N Engl J
Med 2020;382(8):727 - 33. http://dx.doi.org/10.1056/NEJMoa2001 017.

3. Li ZJ, Chen QL, Feng LZ, Rodewald L, Xia YY, Yu HL, et al. Active case finding with case management: the key to tackling the COVID-19 pandemic. Lancet 2020;396(10243):63-70. http://dx.doi.org/10. 1016/S0140-6736(20)31278-2.

4. Zhou L, Wu ZY, Li ZJ, Zhang YP, McGoogan JM, Li Q, et al. One hundred days of coronavirus disease 2019 prevention and control in China. Clin Infect Dis 2021;72(2):332 - 9. http://dx.doi.org/10.1093/ $\mathrm{cid} / \mathrm{ciaa} 725$.

5. Zhang M, Xiao JP, Deng AP, Zhang YT, Zhuang YL, Hu T, et al. Transmission dynamics of an outbreak of the COVID-19 delta variant B. 1. 617. 2-Guangdong Province, China, May-June. China CDC Wkly 2021(27):584 - 6. http://dx.doi.org/10.46234/ccdcw2021.148.

6. Liu FF, Zheng CJ, Wang LP, Geng MJ, Chen H, Zhou S, et al. Interpretation of the protocol for prevention and control of COVID-19 in China (Edition 8). China CDC Wkly 2021;3(25):527-30. http://dx.doi.org/10.46234/ccdcw2021.138.

7. Cheng C, Wang L, Lyu ZQ, Peng B, Li YH, Kong DF, et al. Four COVID-19 cases of new variant B. 1. 351 first emerging in South Africa in Chinese passengers on same flight - Shenzhen, China, January. China CDC Wkly 2021(8):175-7. http://dx.doi.org/10. 46234/ccdcw2021.049.

8. Chen FJ, Li BS, Hao P, Song Y, Xu WB, Liu NK, et al. A case of new variant COVID-19 first emerging in South Africa detected in airplane pilot - Guangdong Province, China, January 6, 2021. China CDC Wkly 2021;3(2):28 - 9. http://dx.doi.org/10.46234/ccdcw2021.007.

9. Qi SX, Zhao X, Hao P, Liu NK, Gao GF, Song Y, et al. Two reemergent cases of COVID-19 - Hebei Province, China, January 2, 2021. China CDC Wkly 2021;3(2):25-7. http://dx.doi.org/ $10.46234 / \mathrm{ccdcw} 2021.006$.

10. Xin HL, Liang JW, Jiang FC. A hospital superspreading event of COVID-19 - Qingdao City, Shandong Province, China, 2020. China CDC Wkly 2020;2(34):655-7. http://dx.doi.org/10.46234/ccdcw 2020.162 . 